\title{
Parametric 3d wind loading on hemispheric dome structures
}

\section{Parametric $3 \mathrm{~d}$ wind loading on hemispheric dome structures}

\author{
Felipe Tavares da Silva \\ Federal University of Bahia, Brazil \\ Faculty of Architecture \\ felipe.tavares@ufba.br
}

\begin{abstract}
Within the visual programming platforms in parametric design, it has not yet available with effective integration the CFD (Computational Fluid Dynamics) simulation systems. This coupling would be particularly useful in relation to the modeling of structures subjected to wind loads in a parametric and algorithmically programmed scenario. It is proposed in this work a parametric modeling of the distribution of wind loads on the surface of a hemispherical dome structure surface. From a combination of wind speed, internal and external pressure coefficient, dimensions of the building, topography and roughness of the terrain. It were defined the magnitude and direction of a field of distributed normal forces on the surface of the hemispherical dome and some results were obtained.
\end{abstract}

Keywords: Wind loading; hemispheric dome; thin shell; grid shell; structures

\section{Introduction}

Within the visual programming platforms in parametric design, it has not yet already available with effective integration the CFD (Computational Fluid Dynamics) simulation systems. This coupling would be particularly useful in relation to the modeling of structures subjected to wind loads in a parametric and algorithmically programmed scenario. It is proposed in this work a parametric modeling of the distribution of wind loads on a hemispherical dome structural surface.

In structural design must be taken into account the effects of mechanical actions due to wind on buildings. The Brazilian standard provides some parameters in your statement on the calculation of wind loads. This Brazilian standard is called NBR 6123 (1988) wind load on building structures, presents an external pressure coefficient isobaric lines for unidirectional flow of wind (Figure 1).

The Eurocode 1: Actions on Structures (1991) gives similar view on wind load when comparing with the Brazilian Standard, as well as other standards and references in this theme (Melaragno,1991; Farshad,1992; Jawad,2004). All these references gives a expression that relates de wind pressure to wind velocity, considering some aspects of geographical, topographical, or geometrical nature. Additionally, in dependency of the building shape these wind models loads the building surfaces on overpressure or suction.

\section{Brazilian Standard external pressure coefficient isobaric lines for domes}

The Brazilian standard for wind load on buildings calculations gives graphical information about the external coefficient pressure due to wind action. In Figure 1 it can be observed its overpressure zones, with coefficients with positive sign; and suction areas, with coefficients with negative sign. It is also noted that the top of this dome concentrates the highest intensity of external suction pressure coefficient. In direct incidence to windward, was observed a maximum coefficient of 0.6. In the leeward it can be observed a sparse representation of numeric values up to -0.2 suction coefficient values.

Once considered the importance of knowing the limitations of construction solutions, certainly there will be opportunity for answers in parametric models.

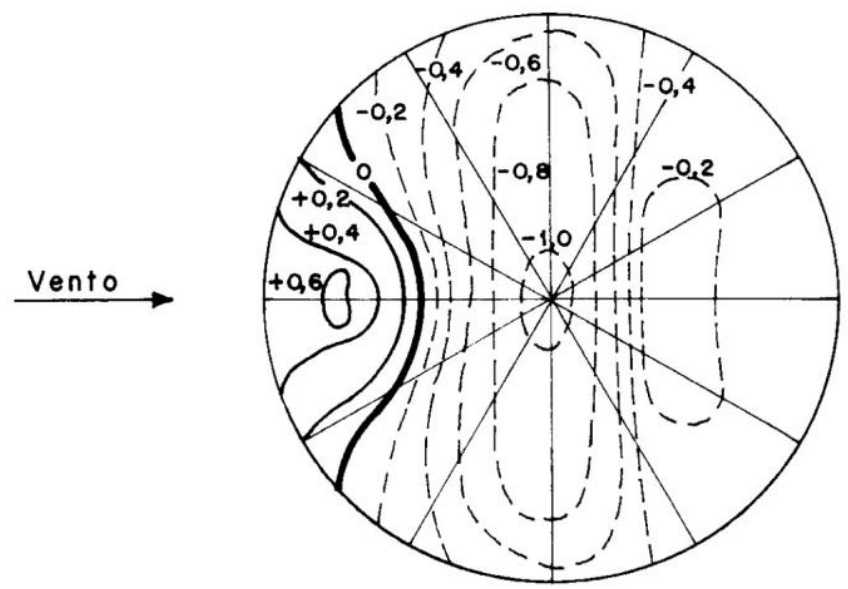

Figure 1: External coefficient isobaric lines diagram for hemispherical building shape in accord with NBR 6123 (1988) standard.

The wind force applied on the surfaces $q_{w s}$, according to NBR6123 (1988) is given by the product of the wind dynamic pressure $q_{d}$ by the difference between the internal and external pressure (Eq. 1).

$q_{w s}=\left(c_{p e}-c_{p i}\right) \cdot q_{d}$ 
The $c_{p e}$ external coefficient pressure for domes is given in Figure 1. The internal pressure coefficient $c_{p i}$ depends on the permeability of the dome winds actions on its surface. This same standard simplifies appointing permeability $c_{p i}=0$ for cases of total impermeability and $c_{p e}=-c_{p i}$ for cases of surface wind permeability above $30 \%$.

This dynamic pressure $q_{d}$ is an estimated quantity of wind pressure in function of geographical, topographical an even the dimension of the building analyzed. With this methodology it can be evaluated in simplified manner the wind mechanical action on a hemispherical dome.

\section{Wind tunnel modeling simulation}

One of the ways to determine the force that the wind applies on building structures is the wind tunnel test. In structural studies of high buildings and complex geometry is reasonable to experience the physical wind tunnel test or simulate it computationally. The ASCE standard for wind tunnel testing testing for buildings and other structures (2012) or the basic CFD reference book by Anderson (1995) are good references for this subject although don't give a direct application of CFD simulations on building structures.

\section{CFD simulation experience over hemispheric dome geometry}

Using the Autodesk Flow Design Software wind tunnel simulation tool, applied on a hemispherical smooth surface, it can be observed the graphic wind velocity flow pattern. The intensity of velocity around the hemispheric dome surface was observed at the top and lateral view, respectively, at middle horizontal and vertical flow plan sections. These graphics consist of photography moment of the transient simulated wind flow and its shown in figures 2 and 3.

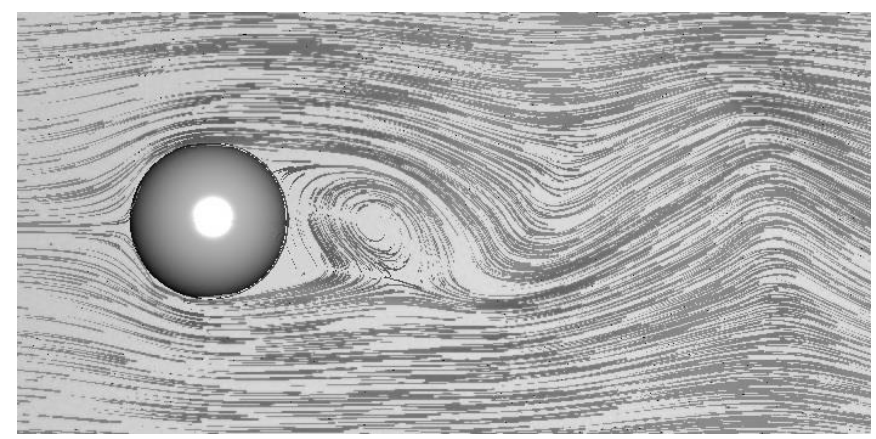

Figure 2: Top view of the hemisphere geometry and the middle height wind flow velocity plan pattern by wind tunnel simulation in a left to right flow sense.

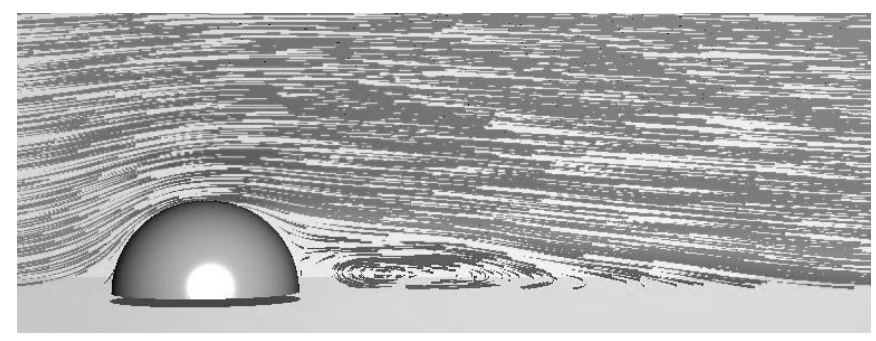

Figure 3: Side view of the hemisphere geometry and the middle point wind flow velocity plan pattern by wind tunnel simulation in the left to right flow sense.
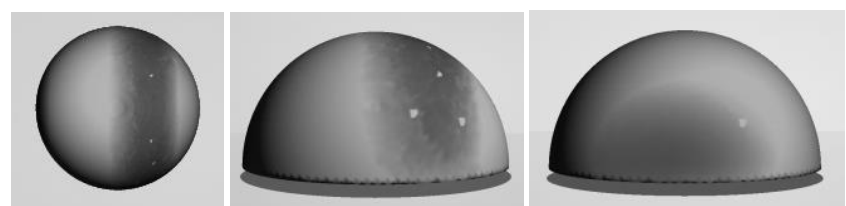

Figure 4: In left to right order: Top, lateral and frontal view of pressure level distribution graphic from a wind tunnel simulation over a hemispherical geometry structure.

It can be observed in those figures the formation of circular vortices in the leeward of the hemispherical surface and in this region the wind acts on a lower velocity flow. Also, it can be seen that in middle lateral and top of the surface has a higher velocity.

In figure 3 is shown the superficial pressure on the hemispheric surface obtained of wind tunnel simulation. It can be observed that it has some similarity when compared with the external coefficient isobaric lines graphic from NBR6123 Brazilian wind load standard.

\section{CFD simulation experience over gridshell geodesic dome geometry}

It can be domes designed structures in thin smooth shell, very close to a smooth hemispherical surface shape, as well as dome structures in trussed gridshells or the peculiar form of geodesic domes.

The characteristic of the faceted geodesic gridshells domes do not present the same aerodynamic behavior as in the case of hemispherical ones. It can be seen this from the CFD simulation on the geodesic dome shape surface (Figures 5,6 and 7).

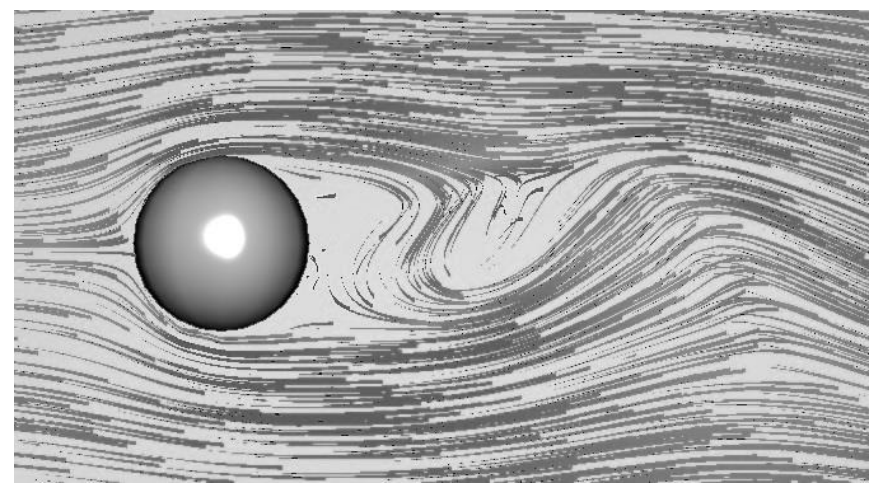

Figure 5: Top view of the geodesic geometry and the middle height wind flow velocity plan pattern by wind tunnel simulation in left to right flow sense.

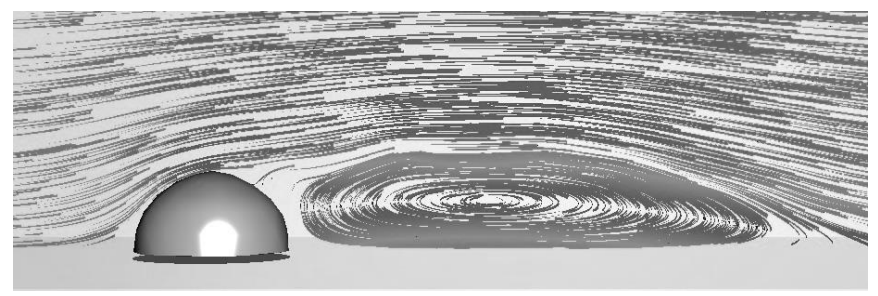


Figure 6: Side view of the geodesic geometry and the middle point wind flow velocity plan pattern by wind tunnel simulation in the left to right sense.
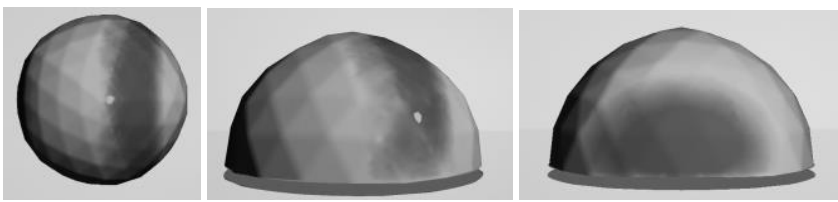

Figure 7: In left to right order: Top, lateral and frontal view of pressure level distribution graphic from a wind tunnel simulation over a geodesic dome geometry structure.

It can be observed that the wind velocity pattern flow on geodesic dome shape is more chaotic and presents bigger vortices at the leeward. The surface pressure distribution of these two types of dome surface shapes showed a similar pattern, as it can been seen comparing figures 4 and 7 .

\section{Parametric modeling of wind structural load}

In this section will be described the process of implementing the wind load isobaric lines diagrams graphically over de some surface geometry, in accordance with the NBR 6123 Brazilian wind structural load standard. This parametrically represented external pressure coefficient distribution, was compared with the results of the CFD simulation and used as input data for structural simulation of a geodesic gridshell dome structure.

\section{External pressure coefficients distributed on hemispherical surface modeling}

The vector field modeling process with the intensity and direction of the wind external pressure coefficients on the surface began with the image stamping Figure 1 on the hemispherical surface. Over this plane isobaric lines figure was drawn up parametric curves through the use of a circle and opened NURBS and chamfered rectangles to model parametrically the isobaric lines diagrams (Figure 8). This task consisted of subdivide de base circle of a considered hemisphere as the starting and ending point of the open curves isobaric lines. For the closed curves it was also considered a straight line for central references of the rectangles. This process was made in a simplified form limited to the precision on CAD zoom and by the definition of scanned image of Figure 1. This graphic parametric information was scaled and linked to a hemispherical surface to obtain this $3 \mathrm{D}$ distribution of wind pressure coefficient.

After parametrically model the flat isobaric contours, a hemispherical surface was created with the goal of stamp these contours on it (Figure 9).

In parallel, it has also modeled a geodesic half icosahedron mesh to obtain the geodesic gridshell bars that were used to model the structural geometry that was simulated (Figure 9). This geodesic geometry was generated with the help of the Weaver Bird Grasshopper's add-on. This is a built-in add-on that generates automatically a icosahedrons by defining the plane and the reference radius, and this mesh was refined by one level. To get only the upper part of the icosahedrons to obtain the geodesic dome, was selected only the mesh panels which has the coordinate $\mathbf{Z}$ of its center with values greater than zero.

Continuing, the plan parametric isolines modeled was projected on the hemispherical shape to obtain the external pressure coefficient 3D distribution from the Brazilian wind load standard by a normal surface vector field (Figure 10).

A built-in Grasshopper feature component was used to obtain the geodesic distance and thereby determine the distance of a point on the hemispherical surface to the nearest isobaric line. Thus, at the points on the dome surface mesh was determined the magnitude and direction of the vector representing the value of the external pressure coefficient at this point.

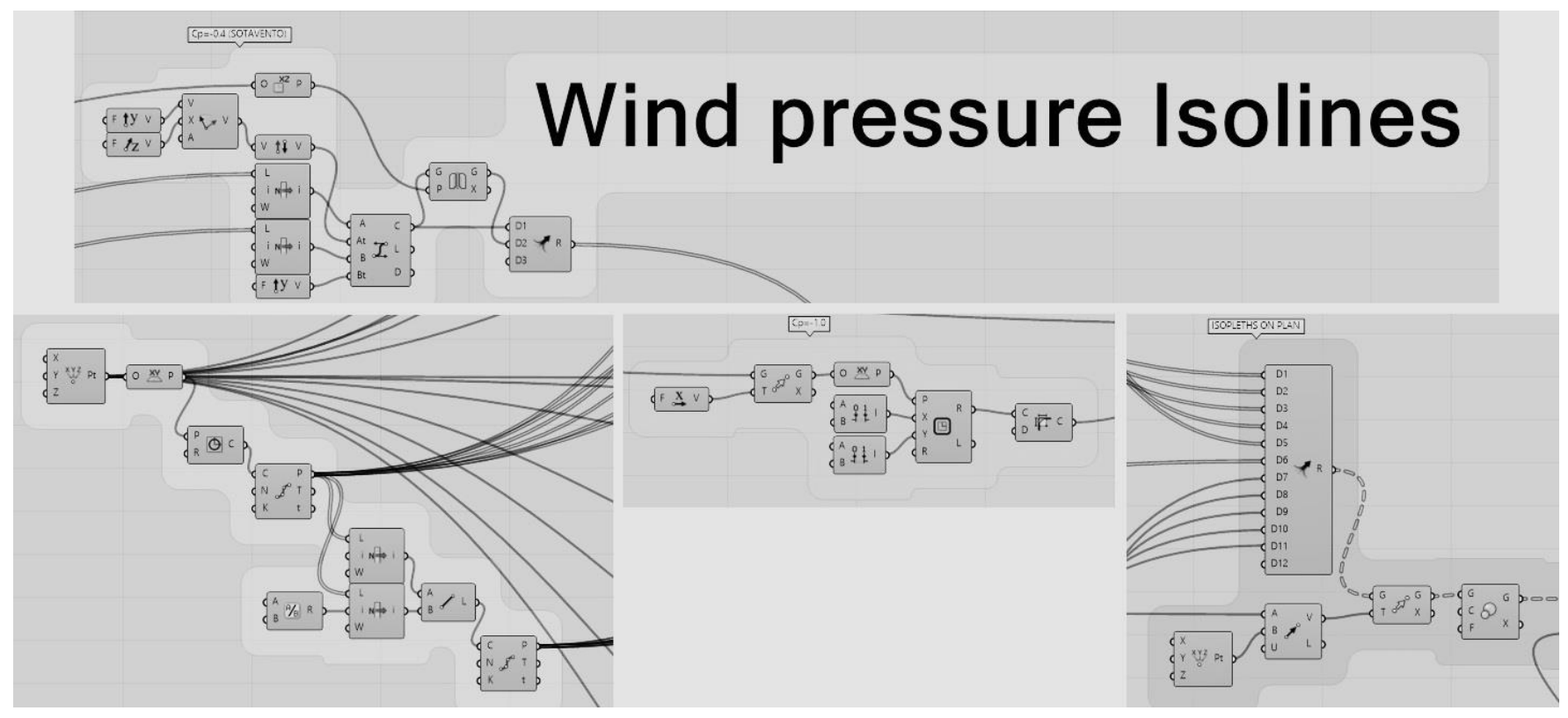

Figure 8: Excerpt of the proposed code with the parametric drawing of isobaric lines of external pressure coefficient. 


\section{Gridshell mesh generation}

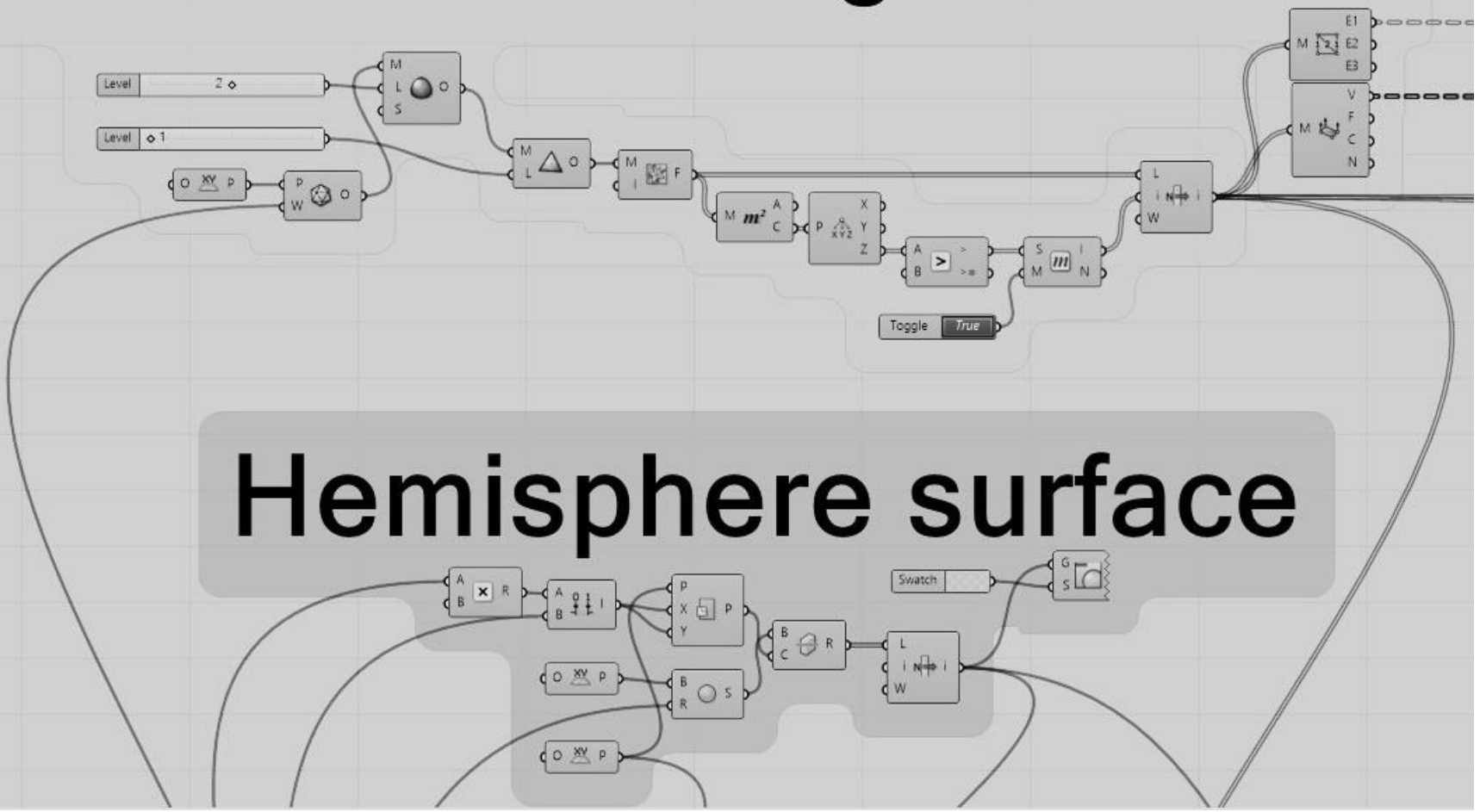

Figure 9: Excerpt of the proposed code generation: the hemispheric and geodesic shapes

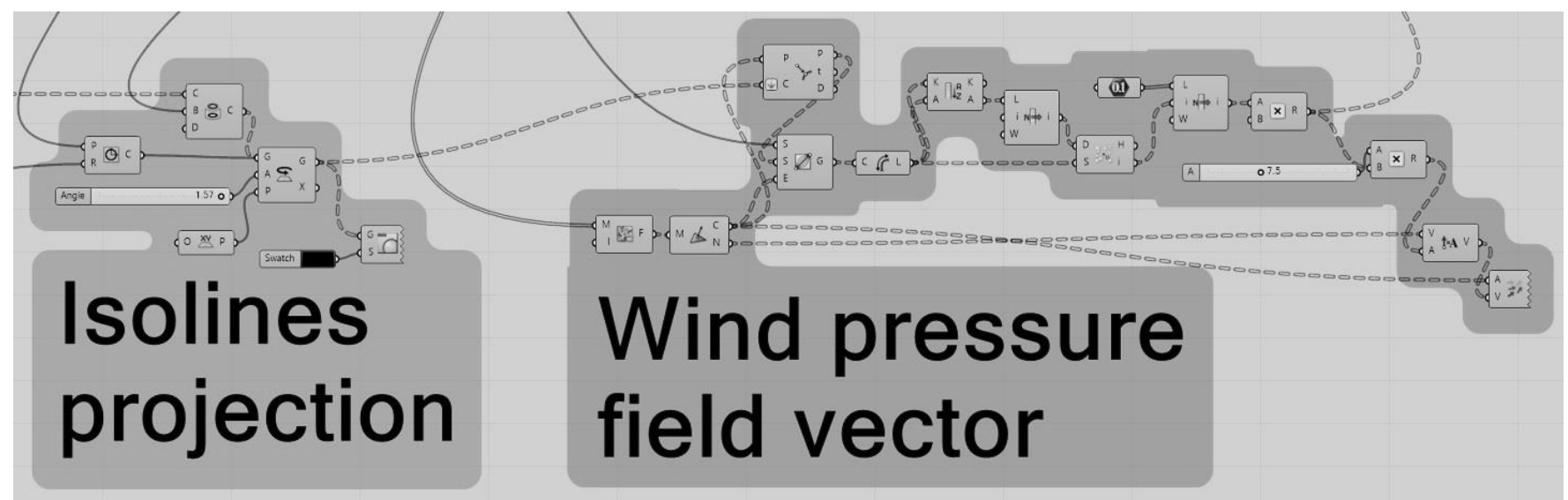

Figure 10: Excerpt of the proposed code generation: isobaric lines projection; creation of the vector field representing the wind pressure distributions.

\section{Applying the wind load on a structure model}

Applying this process at a distribution points on the surface, was obtained then a vector field that represents the distribution of external wind pressure coefficients this geometry according to NBR 6123 standard, with the excerpt of the code tha does it shown in Figure 10.

With the help from the Karamba Structural Modeling add-on for Grasshopper, from Weaver Bird's icosahedrons geodesic gridshell mesh and generated vector field, the structural modeling was made. The Karamba add-on is package with a group of components for Grasshopper environment with the ability to perform structural modeling and these can be designed so that they are parametrically attached to the geometry. This allows for example to perform a structural form finding using search algorithms for design optimized settings.

Figure 11 shows a excerpt of the proposed algorithm code to generate the structural geodesic dome gridshell model.

In this structural modeling, the vector field with the direction and intensity of the external pressure coefficient was used to 
SIGraDi 2016, XX Congress of the Iberoamerican Society of Digital Graphics

9-11, November, 2016 - Buenos Aires, Argentina

calculate the wind load on the faces of geodesic gridshell.

The algorithm automatically calculated the nodal equivalents wind forces in the gridshell structure nodes, due to those face distributed wind load.

In figure 12 it is shown an full overview of the algorithm implemented in Grasshopper environment. It can be seen in this figure the connection between the sections shown in the preceding figures.

It was considered structural bars with tubular steel sections hinged at its ends. The supports were considered in all lower geodesic gridshell nodes.

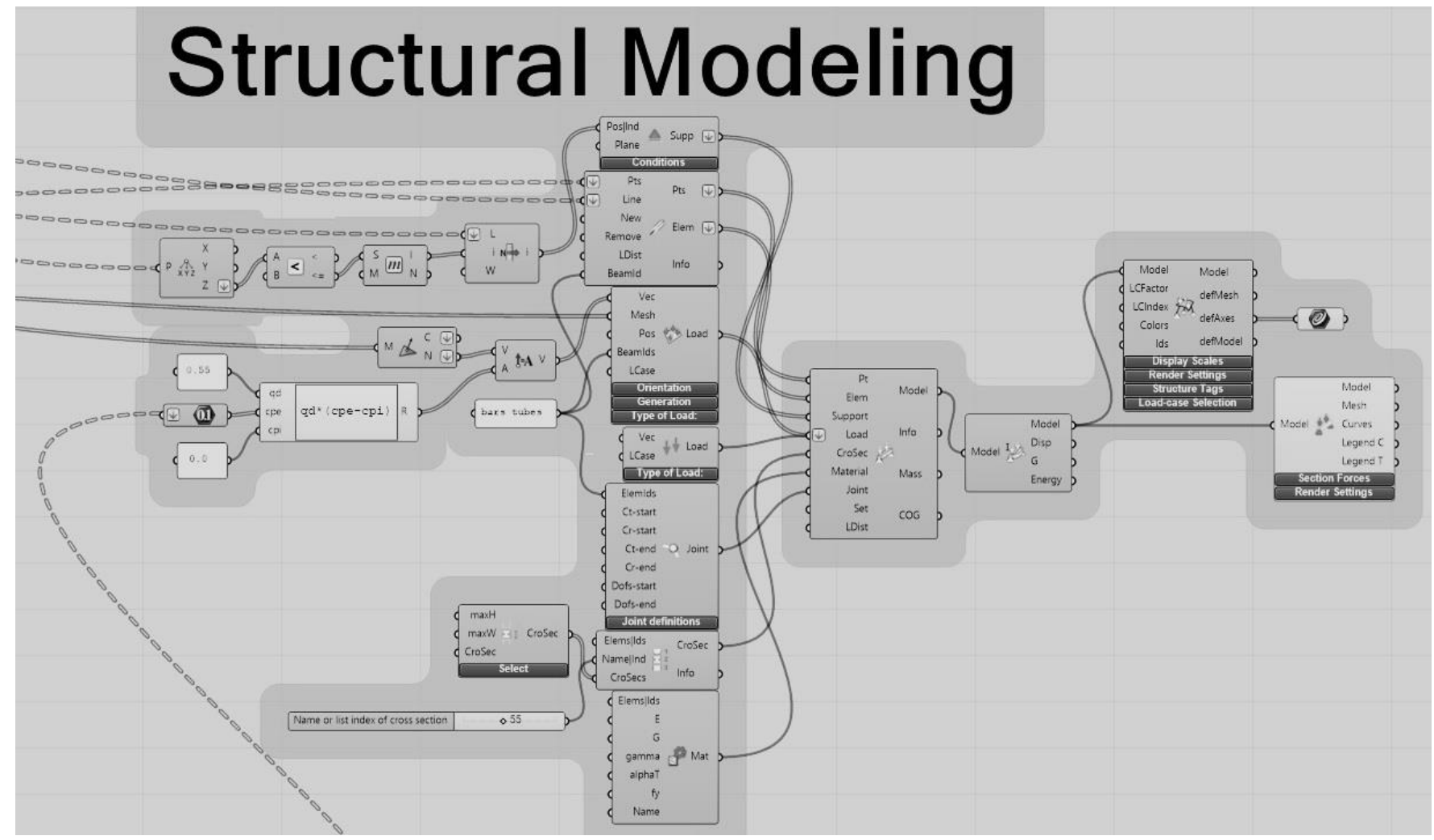

Figure 11: Excerpt of the proposed code generation: structural modeling of geodesic gridshell dome submitted to the proposed wind load.

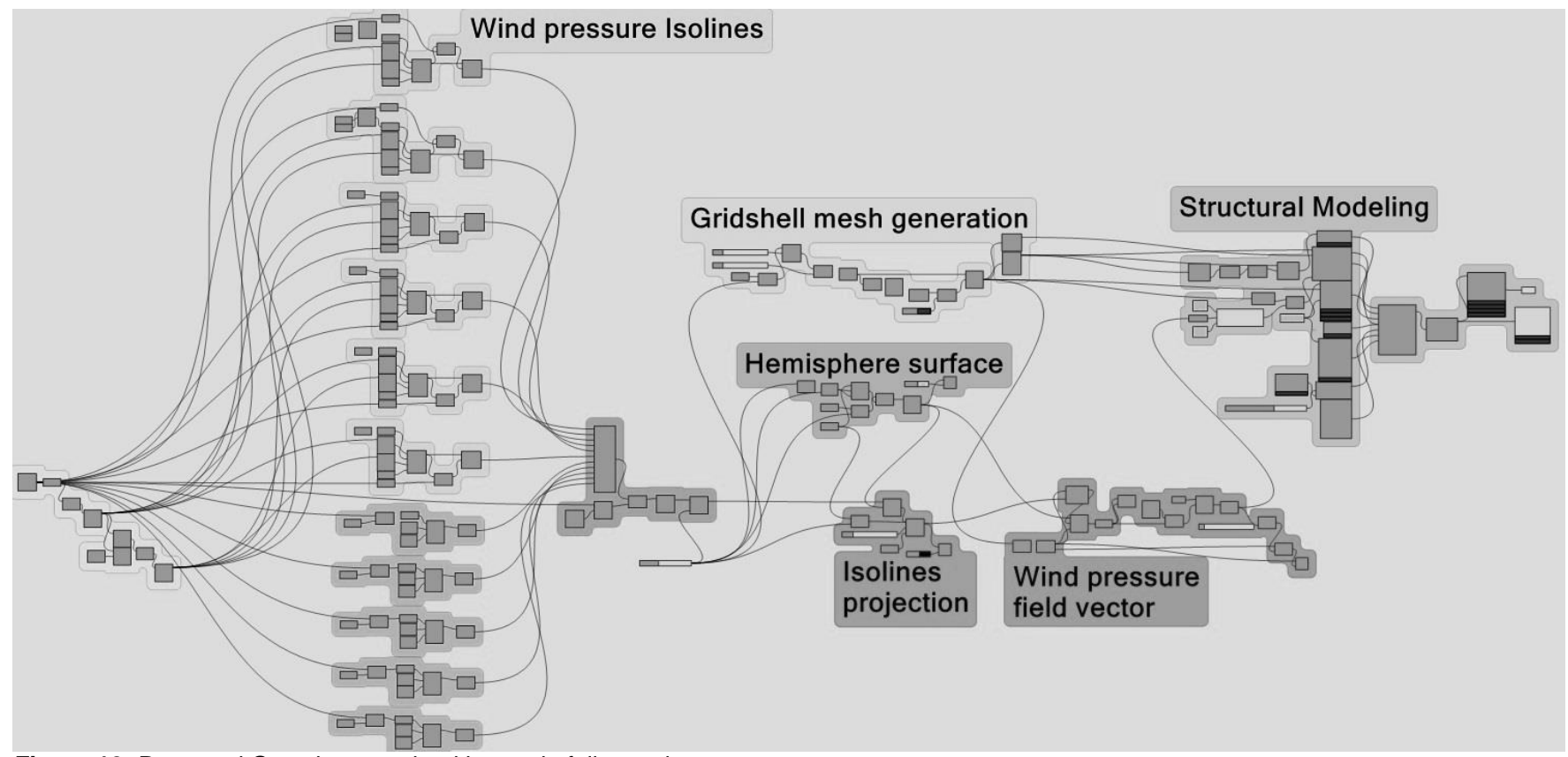

Figure 12: Proposed Grasshopper algorithm code full overview 


\section{Results}

In figure 13 its show the result of the projection the isobaric lines and the vector field indicating the intensity and direction of wind external pressure coefficient on hemispheric shape.

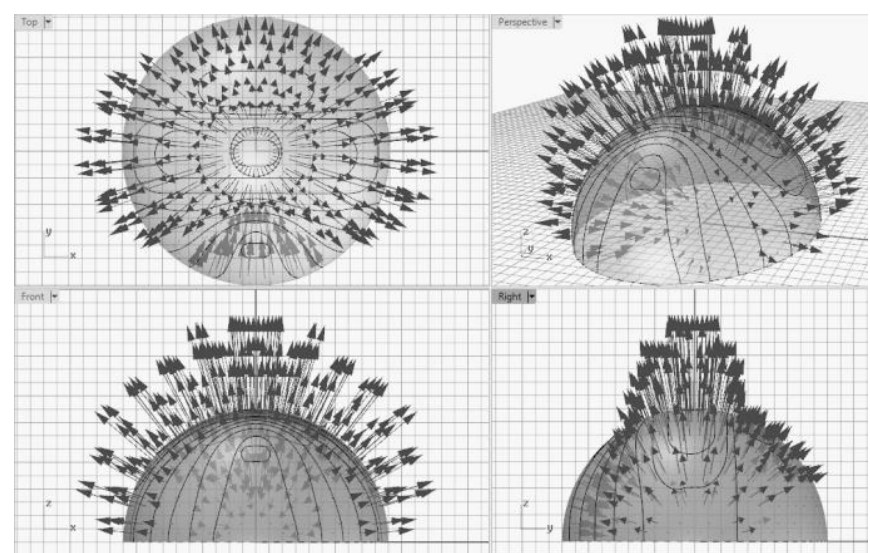

Figure 13: External pressure coefficient vector field: from top left, in clock-wise sense, top; perspective; frontal; and right view.

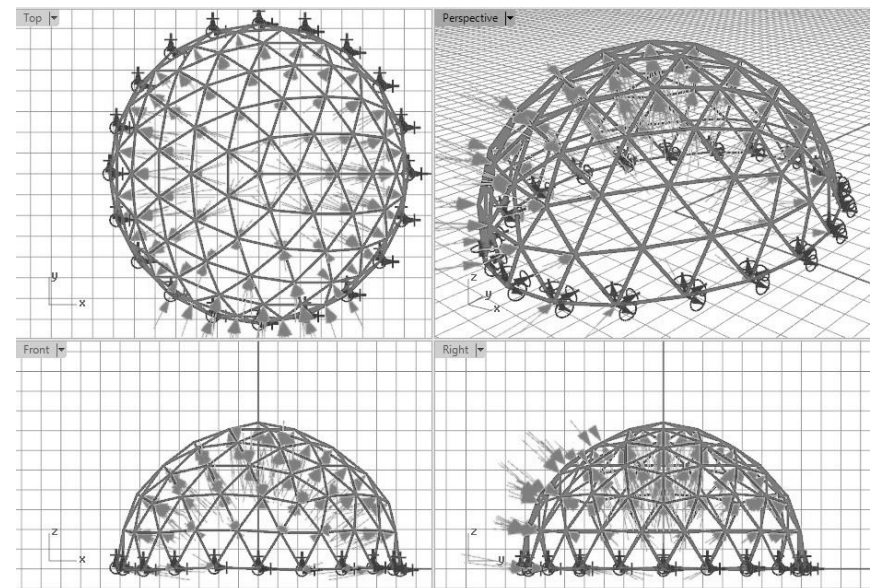

Figure 14: Geodesic gridshell structural modeling: from top left, in clock-wise sense, top; perspective; frontal; and right view.

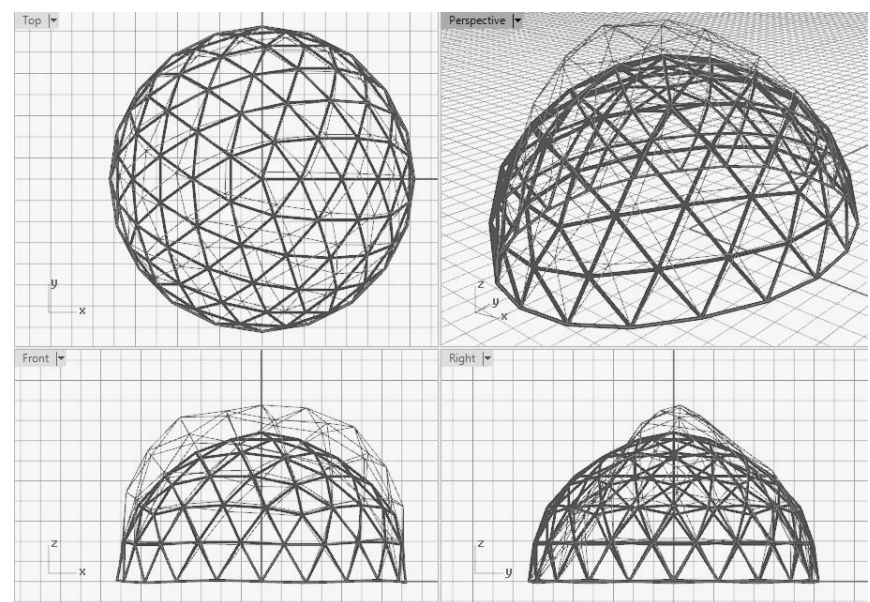

Figure 15: Geodesic gridshell structural deformed shape: from top left, in clock-wise sense, top; perspective; frontal; and right view.

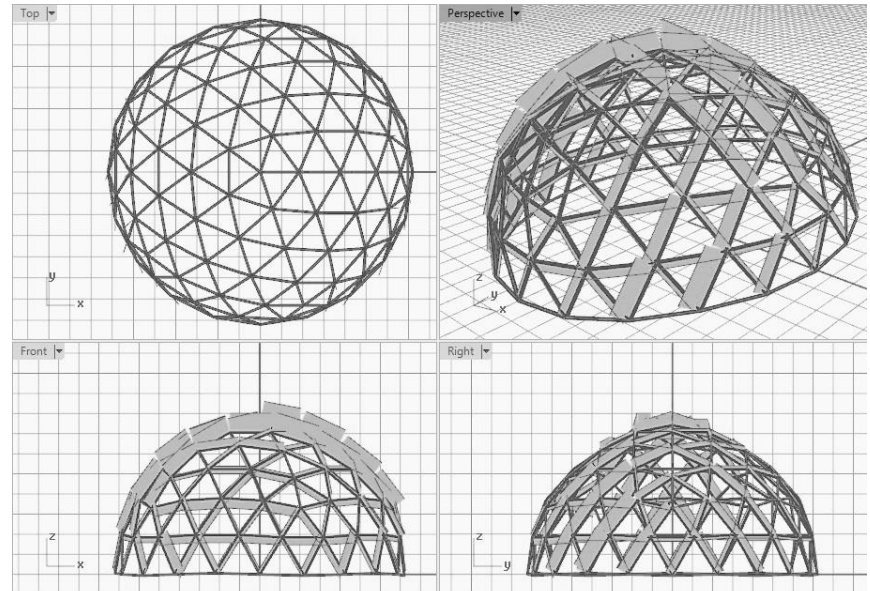

Figure 16: Geodesic gridshell axial forces diagram: from top left, in clock-wise sense, top; perspective; frontal; and right view.

This section also shows the geodesic gridshell geometry modeling through Karamba and their respective results of displacement and axial load in the bars.

In a simplified manner, was also compared pressure on the dome surface calculation results by indications from Brazilian standard against the results observed in the wind tunnel simulation on hemispherical and geodesic domes.

In figure 14 it is shown the geodesic gridshell geometric model with bars, supports and the proposed wind load. In figure 15 and 16 it is shown, respectively, the bars deformed shape and axial forces.

Besides the structural results, also it makes interesting show a comparison of the extreme values of wind load pressure on the surface of the domes. The values of Brazilian standard wind load superficial pressure and the wind tunnel simulation experiments results are shown in Table1.

Table1: Surface pressure extremes values wind loads on domes structures in $\mathrm{KN} / \mathrm{m}^{2}$ associated with wind velocity in $\mathrm{m} / \mathrm{s}$.

\begin{tabular}{|llll|}
\hline Method & velocity & minimal & maximum \\
\hline NBR 6123 & 10 & -0.06 & +0.04 \\
\hline standard & 30 & -0.55 & +0.33 \\
\hline & 50 & -1.53 & +0.92 \\
\hline & & & \\
\hline Hemispheric & 10 & -0.10 & +0.06 \\
\hline CFD & 30 & -0.90 & +0.60 \\
\hline & 50 & -2.50 & +1.60 \\
\hline & & & \\
\hline Geodesic & 10 & -0.05 & +0.06 \\
\hline CFD & 30 & -0.80 & +0.60 \\
\hline & 50 & -2.50 & +1.50 \\
\hline
\end{tabular}

It was not considered any modification of the basic wind velocity value by topographical characteristics in the calculation of extremes values wind loads on Table1. 


\section{Discussions}

In any case, although it can be seen a possible divergence between the wind load pressure models on the Brazilian standard and CFD simulations. Although the vector field of external pressure coefficient proposed has brought consistent results from the point of view of structural modeling.

Indeed, the level of the wind loads observed at this structural setup and shape it was very low. If it is considered light and strength material, it can be possible to build larger domes for roof over smaller or larger areas.

However, this work shows a great lack of sophistication in parametric tools at this time when it comes to CFD simulations and CAD models coupled.

Including the long-standing since the last update, the Brazilian standard for wind load is shown fairly rudimentary when compared to CFD technology. It would be quite interesting when it will update to contain information about the methodologies of modeling and analysis about applying CFD to determine the wind forces on the buildings structures.

It was found that there is indeed a difference between the wind flow patterns when the hemispherical dome has a smooth or faceted surface as in the case of the thin shell and geodesic domes, respectively. It was observed that at $30 \mathrm{~m} / \mathrm{s}$ wind speed, at a tunnel CFD simulation, the wind speed near the surface is around $10 \%$ more faster in the geodesic dome case than in the smooth hemispherical. In relation to the wind pressure on the surface it was observed almost the same level of extreme pressure values.

\section{References}

Anderson, J. D. (1995). Computational Fluid Dynamics. Singapore: McGraw-Hill Books.

Eurocode 1: Actions on structures (1991).

Farshad, M. (1992). Deslgn and analysis of shell structures. Springer.

Jawad, M. H. (2004). Design of plate and shell structures. American Society of Mechanical Engineering.

Melaragno, M. G. (1991). Introduction to shell structures: the art and science of vaulting. Van Nostrand Reinhold.

NBR 6126 (1988). Forças devidas ao vento em edificações. Rio de Janeiro: ABNT-Associação Brasileira de Normas Técnicas. 